University of Wollongong

Research Online

Faculty of Informatics - Papers (Archive)

Faculty of Engineering and Information

Sciences

August 2000

\title{
Shape-VQ-based lossless hybrid ADPCM/DCT coder
}

J. Wang

University of Wollongong

F. Naghdy

University of Wollongong, fazel@uow.edu.au

Follow this and additional works at: https://ro.uow.edu.au/infopapers

Part of the Physical Sciences and Mathematics Commons

\section{Recommended Citation}

Wang, J. and Naghdy, F.: Shape-VQ-based lossless hybrid ADPCM/DCT coder 2000.

https://ro.uow.edu.au/infopapers/87

Research Online is the open access institutional repository for the University of Wollongong. For further information contact the UOW Library: research-pubs@uow.edu.au 


\title{
Shape-VQ-based lossless hybrid ADPCM/DCT coder
}

\begin{abstract}
The discrete cosine transform (DCT) has been shown as an optimum encoder for sharp edges in an image (Andrew and Ogunbona, 1997). A conventional lossless coder employing differential pulse code modulation (DPCM) suffers from significant deficiencies in regions of discontinuity, because the simple model cannot capture the edge information. This problem can be partially solved by partitioning the image into blocks that are supposedly statistically stationary. A hybrid lossless adaptive DPCM (ADPCM)/DCT coder is presented, in which the edge blocks are encoded with DCT, and ADPCM is used for the non-edge blocks. The proposed scheme divides each input image into small blocks and classifies them, using shape vector quantisation (VQ), as either edge or smooth. The edge blocks are further vector quantised, and the side information of the coefficient matrix is saved through the shape-VQ index. Evaluation of the compression performance of the proposed method reveals its superiority over other lossless coders.

\section{Keywords}

adaptive modulation, differential pulse code modulation, discrete cosine transforms, image coding, modulation coding, transform coding, vector quantisation

\section{Disciplines}

Physical Sciences and Mathematics

\section{Publication Details}

This paper originally appeared as: Wang, J \& Naghdy, F, Shape-VQ-based lossless hybrid ADPCM/DCT coder, IEEE Proceedings - Vision, Image and Signal Processing, August 2000, 147(4), 313-317. Copyright IEEE 2000.
\end{abstract}




\title{
Shape-VQ-based lossless hybrid ADPCM/DCT coder
}

\author{
J.Wang and G.Naghdy
}

\begin{abstract}
The discrete cosine transform (DCT) has been shown as an optimum encoder for sharp edges in an image (Andrew and Ogunbona, 1997). A conventional lossless coder employing differential pulse code modulation (DPCM) suffers from significant deficiencies in regions of discontinuity, because the simple model cannot capture the edge information. This problem can be partially solved by partitioning the image into blocks that are supposedly statistically stationary. A hybrid lossless adaptive DPCM (ADPCM)/DCT coder is presented, in which the edge blocks are encoded with DCT, and ADPCM is used for the non-edge blocks. The proposed scheme divides each input image into small blocks and classifies them, using shape vector quantisation (VQ), as either edge or smooth. The edge blocks are further vector quantised, and the side information of the coefficient matrix is saved through the shape-VQ index. Evaluation of the compression performance of the proposed method reveals its superiority over other lossless coders.
\end{abstract}

\section{Introduction}

In electronic medical recording, the storage and transmission requirements of image data necessitate some form of compression. Thus reversible compression techniques and progressive transmission methods aimed at reducing the overhead incurred in transmitting full resolution images have been the subject of intensive research over the last decade.

Image data are usually modelled as a stationary, highly correlated process. However, this assumption is rarely true, in practice, because typical images contain edges of various lengths and orientations. In those correlated areas where the assumption of stationarity holds, differential pulse code modulation (DPCM) can perform very well. At the edges, a more suitable model of the image is needed. Discrete cosine transform (DCT) is accepted as the best approximation of an optimum transform, the KarhunenLoeve transform (KLT), for highly correlated sources. Recently, it has been shown in [1] that DCT is also a zero-DC constrained near optimum transform for encoding step edge information.

A typical image contains segments that can be described variably as edge and smooth regions. Obviously, these segments should be modelled differently, so that an image coder can adaptively take advantage of this segmentation. The strategy adopted in the coder presented in this paper is to partition the image into edge areas that can be encoded by DCT, and the other areas that can be encoded by ADPCM.

Encoding images through different modules is by no means a new idea. However, a special kind of VQ, shape-

(C) IEE, 2000

IEE. Proceedings online no. 20000399

DOI: 10.1049 /ip-vis:20000399

Paper first received 20th April and in revised form 6th December 1999

The authors are with the School of Electrical, Computer \& Telecommunication Engineering, University of Wollongong, Northfield Avenue, Wollongong NSW 2500, Australia
VQ, is used in this paper as the classifier. The breakthrough in the classification is due to the ability of the shape-VQ accurately to capture local features inside the image block and provide the encoder with a brief view of the blocks. In further enhances the performance of the DCT used in a lossless way, as the side information is fairly limited. The outstanding performance of the proposed method proves its competence.

\section{Shape VQ-based lossless ADPCM/DCT coder}

\subsection{Shape-VO classifier}

In the proposed coder, the image is partitioned into distinct blocks of $8 \times 8$ pixels, and each block is mean-normalised, using the following equation:

$$
\tilde{f}(i, j)=f(i, j) / \bar{B}
$$

where $\tilde{f}(i, j)$ is the pixel value in the mean-normalised block, $f(i, j)$ is the raw pixel value, and $\bar{B}$ is the block mean.

This process removes the bias of the block, and blocks with similar features can be picked up regardless of their background grey-scale value. The features include boundary, edge and texture in natural image and are characterised by the variance of the pixel value inside the block. As all of these features contribute to the shape of image, this kind of vector is called a shape vector.

There is a choice between an image adaptive or universal VQ scheme. The advantage of using an adaptive VQ derives from the possibility of reducing the distortion associated with the classification. However, the need to transmit the codebook as side information results in a constraint on the size of the codebook. This can negatively impact on the performance of the coder. In using a universal codebook, the constraint of codebook length is removed, but the ability of the codebook adequately to represent the input image can be reduced. A compromised universal codebook with size of 256 is chosen for the simulations reported in this paper.

The codebook is set up by the famous LBG algorithm. The VQ in this stage is thereafter called VQ1. The variance inside the vector is used to determine its classification, 
where each shape block is classified into one of two classes, edge or smooth, by a pre-set threshold $\delta_{T}$.

For systems using adaptive $\mathrm{VQ}, \delta_{T}$ can be chosen adaptively as well. However, this will increase the computational burden considerably. In addition, current $\delta_{T}$ has been chosen through extensive tests, making it optimum for normal images in terms of final decorrelation results. A poorly chosen threshold results in the selection of the 'wrong' encoder for the blocks, which means either ADPCM or DCT is 'overused'. Experimental results have shown that ADPCM alone generates worse results than those obtained with the proposed scheme, and DCT alone, as is widely known, is not suitable for lossless coding; overusing either of them can produce worse compression results for lossless coding. Therefore, in this research, as universal VQ is applied in all the cases, $\delta_{T}$ is also fixed. The optimum $\delta_{T}$ is generated from several images that include normal and medical images. For mammograms (please refer to Section 4), another threshold is used. This improves the performance of the system with a particular group of images, such as a mammogram.

Using a shape vector brings a more accurate classification than is obtained directly with raw image blocks, as the interference from the image background is eliminated through the mean normalisation. This further improves the performance of the universal codebook, as blocks from different pictures with different backgrounds can be compared in a uniform standard.

Tests have been carried out to verify the effectiveness of shape-VQ as a classifier. As variance is a good indication of the concentration of data, it is used to measure the performance of shape-VQ. Two images, 'Lena' and 'Feet', are tested. There is a large difference between the mean of these two images. The mean of the whole image of 'Lena' is around 170, and that of 'Feet' is only about 25 .

In the first test, a threshold $\delta_{T 1}$ is chosen for the variance classifier, so that those blocks in Lena whose variance of raw data is larger than $\delta_{T 1}$ are classified as edge blocks, and the rest are classified as smooth. The threshold $\delta_{T 1}$ is adjusted to make the classification similar to those with shape-VQ. In the shape-VQ classifier, a fixed $\delta_{T}$ is used. When both classifiers are applied to 'Feet', it is interesting to see that all the blocks are recognised as smooth blocks a with variance classifier, which is untrue for the image. It can be seen from the picture in Fig. 1 that there are still many edge components in 'Feet', although it is much smoother than 'Lena'. This kind of misclassification is mainly due to the large difference of the backgrounds of the images.

In the second test, threshold $\delta_{T 1}$ is adjusted to $\delta_{T 1}^{\prime}$ when it is applied to Feet, so that

$$
\delta_{T 1}^{\prime}=\delta_{T} * \frac{\text { mean of 'Feet' }}{\text { mean of 'Lena' }}
$$

However, the result is still not up to the level of shape-VQ. It shows that edge components are very complex in an image. It is very hard to capture them accurately at the whole-image level. Shape-VQ overcomes these difficulties by looking at the edges at block level and comparing them in a same platform. This explains why classifiers using variance within shape-VQ outperform those directly using variance.
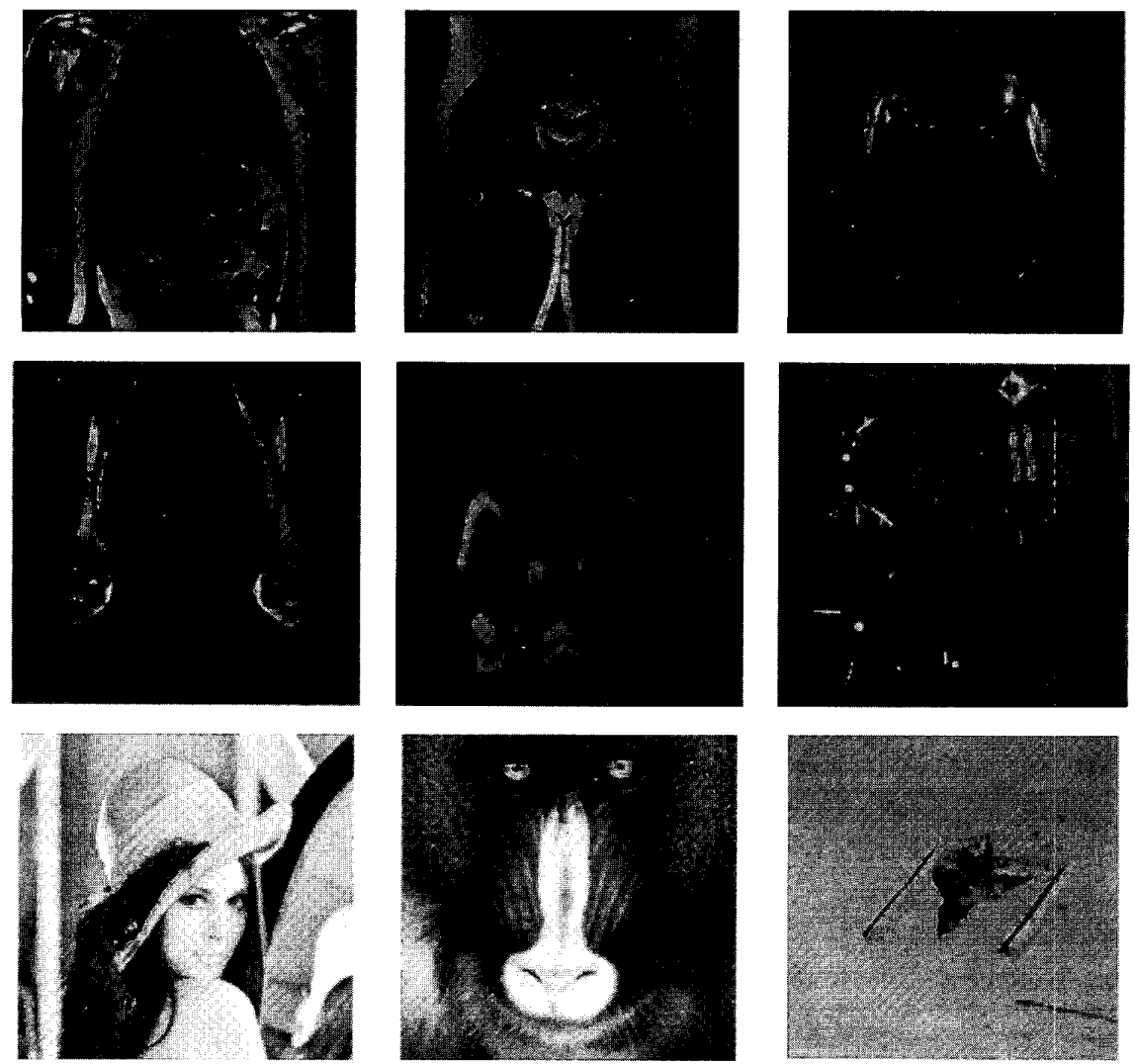

Fig. 1 Mosaic of test images used in simulation

Top (left to right): 'Abdo', 'Pelvis', 'Head' Middle (left to right): 'Feet', 'Skull', 'Lax' Bottom (left to right): 'Lena', 'Baboon', 'Jet' 
Table 1: Results of compression by shape-VQ classifier, direct variance $\left(\delta_{T 1}\right)$ classifier and adjusted variance $\left(\delta_{T 1}^{\prime}\right)$ classifier

\begin{tabular}{|c|c|c|c|c|c|c|c|c|c|}
\hline & \multicolumn{3}{|c|}{ Shape-VO } & & \multicolumn{3}{|l|}{$\delta_{T 1}^{\prime}$} \\
\hline & edge & smooth & entropy & edge & smooth & entropy & edge & smooth & entropy \\
\hline Lena & 196 & 828 & 4.38 & 196 & 828 & 4.38 & 196 & 828 & 4.38 \\
\hline Feet & 89 & 935 & 2.03 & 0 & 1024 & 2.25 & 14 & 1010 & 2.36 \\
\hline
\end{tabular}

The test results are listed in Table 1. Those numbers in Table 1 under 'Edge' and 'Smooth' are the numbers of blocks that are classified as edge or smooth. All classifiers are in the same framework of the proposed ADPCM/DCT coder. The entropies are the first-order entropy of errors.

\subsection{ADPCM module}

A set of prediction parameters of ADPCM are found for each code vector by the two-dimensional Levinson and modified multi-channel version of the Burg algorithm introduced in [2]. Thus the codebook of the shape vector includes three parts, which are the index of the vector, the shape vector and the set of prediction parameters appointed to the vector. This is clearly shown in Fig. 2, where $S$ stands for a smooth block, and $E$ stands for the edge.

When an image block $A$ appears, it is first mean-normalised, and this generates a shape vector $\tilde{A}$. The shape vector is then vector quantised. A code vector is found for $\tilde{A}$ based on the nearest-neighbour rule. If this code vector is assigned as a smooth block, ADPCM will be applied to $A$, and the prediction parameters can also be found in the codebook, which is indicated by the index.

The prediction errors are rounded down to the nearest integer by

$$
\bar{e}=\lfloor e\rfloor
$$

where $(e-1)<\bar{e} \leq e$ and $\bar{e} \in Z$. The final error $\bar{e}$ is transmitted or stored.

\subsection{Shape-VQ-based DCT module}

It has been proved in [1] that the DCT is a good candidate for compressing the edge components in an image. Therefore it is used in this research for decorrelating edge blocks.

To prove the performance of the DCT for edge components, we tested it with edge blocks in the codebook of VQ1. The average mean squared errors (MSEs) between the reversed image blocks and the original ones of the DCT are compared with those of uniform DPCM and ADPCM.

From the results in Table 2, it can be seen that, for edge blocks, the DCT surely outperforms those predictive tech-

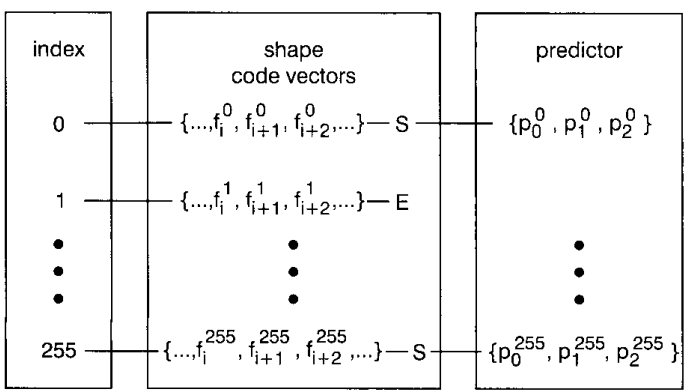

Fig. 2 Codebook of shape- $V Q I$
Table 2: Mean squared errors (MSEs) of three schemes applied to edge blocks

\begin{tabular}{llll}
\hline & DPCM & ADPCM & DCT \\
\hline MSE & 2.96 & 2.73 & 1.94 \\
\hline
\end{tabular}

niques, and it also verifies the conclusion made in [1], which states that the DCT can maximise the energy packing efficiency (EPE), which is equivalent to minimising the MSEs in terms of step response (edge).

It has been reported in [3] that similar shape blocks have close prediction parameter vectors. This is also true for the DCT coefficient matrix. The coefficient matrix of the DCT represents the variation inside the image block. This variation is a sign of the shape and is defined in [4] (p.390) as an abrupt change in the pixel value. Therefore it can be concluded that one shape vector is corresponding to a certain DCT coefficient matrix. Therefore shape-VQ is used to save the complicated calculation in DCT and the side information of the coefficient matrix.

Another universal shape-VQ codebook, VQ2, is set up specifically for the edge blocks. The DCT is then applied to each block in the codebook. The computation of the DCT used in this paper is similar to the lossy coder recommended by JPEG [5]. The resulting matrix is stored and is indicated by the index in the codebook. Therefore the VQ index depicts not only the edge block in the codebook but also the coefficient matrix of this code vector. This is shown in Fig. 3.

During the encoding of images, those blocks labelled as edge components are vector quantised by VQ2. The errors between the original block and the reversed one are calculated and retained as integers through the operator in eqn. 3. Also kept is the index of the block.

From the depiction above, it can be seen that, for smooth blocks, only 8 bits of side information are needed for the whole block, and edge blocks require 8 bits extra for the VQ index. This is all the side information of the proposed scheme.

The whole system is shown in Fig. 4.

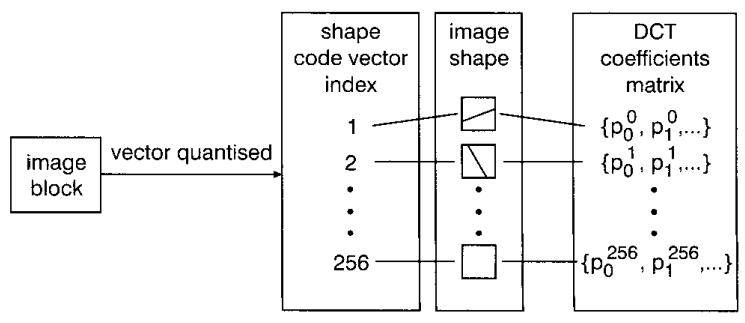

Fig. 3 Proposed shape-DCT/VQ2 encoder 


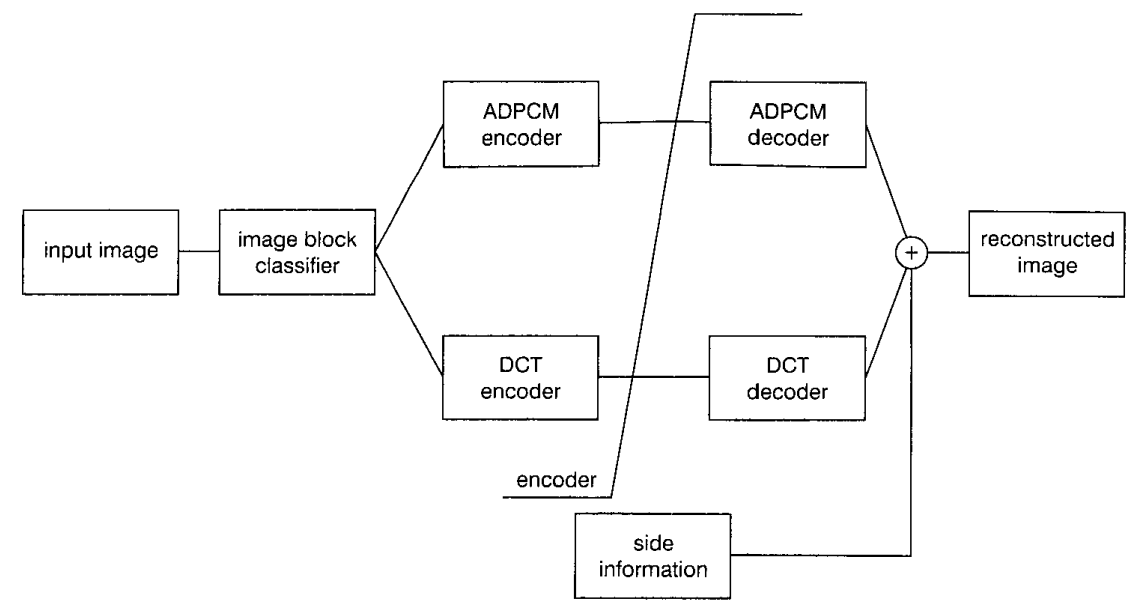

Fig. 4 Block diagram of proposed coder/decoder pair

\section{Results and discussion}

The test images used in assessing the proposed coder include medical images, 'Abdo', 'Feet', 'Head', 'Pelvis' and 'Skull'. In addition, natural images, 'Lena', 'Baboon', 'Jet' and 'Lax', have been used to gauge the performance on such images. All the images are $512 \times 512$ pixels with 256 grey scale. A comparison of the performance of the proposed coder against DPCM, HINT, hybrid coder and CALIC is presented. The results quoted here are the firstorder entropy of the errors plus the bits used for side information. This enables us to compare all the methods fairly and in the same platform. However, the proposed ADPCM/DCT can be interfaced with any entropy coding methods, as the errors are independent of the prediction (redundancy has been removed). The block size of the proposed coder is $8 \times 8$.

The training sets for the codebooks of VQ1 and VQ2 include a wide range of blocks from different images. The images used in the training are different from those used in testing the compression algorithm.

Table 3 compares the bit rates achieved by the proposed coder, DPCM and HINT. They indicate that the proposed

Table 3: Results of lossless compression of images by DPCM, HINT, MAR/VQ and ADPCM/DCT

\begin{tabular}{lllll}
\hline Test image & DPCM & HINT & MAR/NO & ADPCM/DCT \\
\hline Lena & 4.53 & 4.47 & 4.32 & 4.24 \\
Baboon & 6.14 & 5.82 & 5.79 & 5.47 \\
Jet & 3.82 & 3.33 & 3.28 & 3.23 \\
Lax & 5.65 & 5.47 & 5.39 & 5.06 \\
\hline
\end{tabular}

scheme can outperform these well-known methods. The gain in bit rate is attributable to the careful modelling and encoding of the edge blocks.

A multiplicative auto-regressive (MAR) model has been proposed for predictive coding, and good results have been reported when it is applied to lossless compression [6]. A hybrid scheme combining multiplicative modelling and vector quantisation was proposed in [3], and the results presented showed an improvement over [6]. A comparison of the scheme proposed in this paper with results reported in [3] shows that the proposed technique achieves a better performance than a coder based on a multiplicative image model.

The lossless version of the JPEG image compression standard has been compared with the proposed technique [5]. Table 4 shows the bit rate achieved using medical images. These figures (minimum value in bold) can be compared with those of ADPCM/DCT. The proposed technique is seen to outperform the lossless JPEG standard for these medical images.

The newly developed context-based, adaptive, lossless image codec (CALIC) has been reported as efficient in lossless compression in [7]. In this paper, results from CALIC are also compared with those of the proposed coder. In Table 5, performances of these two coders for 'typical' images are presented. Compression results using mammograms from the Digital Mammogram Library of Lawrence Livermore National Laboratory are shown in Table 6. The mammograms, with various spatial resolutions, are all in 16 bits per pixel. Special codebooks are set up for these mammograms. The training set is from the mammograms, and none of the test images is included.

From the results in Table 5, it can be seen that the proposed coder gives a similar performance to that of CALIC, especially in some images with complex scenes,

Table 4: Performance of lossless JPEG on medical images

\begin{tabular}{lllllllll}
\hline Test images & JP1 & JP2 & JP3 & JP4 & JP5 & JP6 & JP7 & ADPCM/DCT \\
\hline Skull & 3.34 & 3.28 & 3.73 & 2.51 & 2.65 & 2.56 & 2.86 & 2.28 \\
Abdo & 5.80 & 5.43 & 6.15 & 5.04 & 5.16 & $\mathbf{4 . 9 3}$ & 5.25 & 4.19 \\
Feet & 2.92 & 2.39 & 3.09 & 2.27 & 2.43 & $\mathbf{2 . 2 3}$ & 2.43 & 2.02 \\
Head & 3.55 & 3.35 & 3.84 & 3.09 & 3.00 & $\mathbf{2 . 9 0}$ & 3.01 & 2.57 \\
Pelvis & 5.02 & 4.52 & 5.32 & 4.23 & 4.32 & $\mathbf{4 . 0 0}$ & 4.36 & 3.41 \\
\hline
\end{tabular}


Table 5: Performance of CALIC and ADPCM/DCT on normal images

\begin{tabular}{lll}
\hline Test image & CALIC & ADPCM/DCT \\
\hline Lena & 4.35 & 4.24 \\
Baboon & 5.68 & 5.47 \\
Jet & 3.07 & 3.23 \\
Lax & 5.13 & 5.06 \\
\hline
\end{tabular}

Table 6: Performance of CALIC and ADPCM/DCT on mammograms

\begin{tabular}{lcc}
\hline Mammogram & CALIC & ADPCM/DCT \\
\hline Afrcc & 2.57 & 2.43 \\
Aflcc & 2.63 & 2.53 \\
Agrcc & 2.37 & 2.25 \\
Aglcc & 2.38 & 2.27 \\
Ahrcc & 2.61 & 2.35 \\
Ahlcc & 2.59 & 2.36 \\
Aircc & 2.27 & 2.26 \\
Ailcc & 2.26 & 2.13 \\
Ajrcc & 2.37 & 2.30 \\
Ailcc & 2.29 & 2.06 \\
\hline
\end{tabular}

Table 7: Running time of CALIC and ADPCM/DCT on test images, where all data are in seconds

\begin{tabular}{lcc}
\hline Test image & CALIC & ADPCM/DCT \\
\hline Lena & 1.35 & 2.55 \\
Baboon & 1.38 & 2.78 \\
Lax & 1.35 & 2.60 \\
Afrcc & 8.89 & 10.90 \\
Aflcc & 9.12 & 11.93 \\
Agrcc & 8.76 & 10.89 \\
\hline
\end{tabular}

such as 'Baboon', as more edge components can be found in this sort of image. This confirms the strong performance of the DCT in decorrelating the edge image blocks. Results from the mammograms further prove this point. Mammogram images are textured, with many strong edge points. This gives the new scheme a good chance to show the shape-VQ's ability to grasp local features and classify accurately the image blocks. The competence of the DCT module in edge components is further confirmed.

The running times of both coders are presented in Table 7. It shows the weakness of the proposed scheme in terms of calculation speed as VQ is included. However, this is a minor penalty for the much more important gain of storage space for medical image archiving, in particular in cases of high-density images such as mammograms.

\section{Conclusions}

The paradigm adopted in the proposed coder can be regarded as segmentation-based. In any given image, it is difficult to find one model that sufficiently describes information inherent in the pixel arrangement. However, by classifying the various arrangements into identifiable segments that can be modelled, the coding task is made easier. This paper uses the DCT as the optimum descriptor of an ideal step edge information, along with the shape VQ-based classifier and quantiser to improve the coding performance of ADPCM. The results presented show the efficacy of this paradigm.

\section{References}

1 ANDREW, J., and OGUNBONA, P.: 'On the step response of the DCT', IEEE Trans. Circuits Syst. II, Analog Digit. Signal Process. 1997,44 (3) pp $260-262$

2 KUDUVALLI, G., and RANGAYYAN, R.: 'Performance analysis of reversible image compression techniques for high-resolution digita teleradiology', IEEE Trans. Med. Imaging, 1992, 11, (3), pp. 430-445

3 OGUNBONA, P., WANG, J., and NAGHDY, G.: 'Hybrid predictive' VQ lossless image coding', Electron. Lett., 1995, 31, (6), pp. 441-442

JAIN, A.: 'Fundamentals of digital image processing' (Prentice-Hall, Inc., Eastwood Cliffs, New Jersey, 1989)

WALLACE, G.: 'The JPEG still picture compression standard', IEEE Trans. Consum. Electron., 1992, 38, (1), pp. xviii-xxxiv

6 DAS, M., and BURGETT, S.: 'Lossless compression of medical images using two-dimensional multiplicative auto-regressive models', IEEE Trans. Med. Imaging, 1993, 12, pp. 721-726

7 WU, X., and MEMON, N.: 'Context-based, adaptive, lossless image coding', IEEE Trans. Commun., 1997, 45, (4), pp. 437-444 